\title{
Research on choices of methods of internet of things pricing based on variation of perceived value of service
}

\author{
Wei Li, Lijun Mei, Kai Nie \\ College of Economic and Trade, Hunan University (China) \\ liweibncs@,hnu.edu.cn,limei@,hnu.edu.cn, knie@,bnu.edu.cn
}

Received November 2012

Accepted February 2013

\section{Abstract:}

Purpose: With the rapid progress of Internet of Things technology, the information service of IoT has got unprecedented development, and plays an increasingly important role in real life. For the increasing demand of information service, the pricing of information service becomes more important. This paper aims to analyze the strategic options and payoff function between information provider and intermediaries based on Stackelberg game. Firstly, we describe information service delivery method based on the Internet of Things specific function. Secondly, we calculate the consumer demand for the information service. Finally, we explain two kinds of strategic options by the game theory, and then discuss the optimal pricing method of information services based on profit maximization.

Design/methodology/approach: To achieve this objective, Considering the consumer perceived value of Internet of Things Service changing, we establish a Stackelberg model in which the supplier is the leader followed by the middleman. Then, we compare the advantages of using individual pricing with that of bundling pricing.

Findings: The results show that whether information providers adopt bundling pricing strategy or individual pricing strategy depends on the cost of perception equipment, if information 
providers want to adopt individual pricing strategy, the variation of consumers' perception value of information services must meet certain conditions.

Research limitations/implications: the providers make price for the information service, in addition to continuously improve the quality of information service, it also devotes resources to tapping and understanding market information, such as the sensor device price, the variation of perception value of information services and so on, so as to create competitive advantage. This paper is just a preliminary model, it does not take into account the effect of mixed bundling.

Originality/value: In this research, a new model for price information service with the game theory is proposed. To the authors' knowledge, it is the first time to study the pricing of information service with the game theory, It discuss the impact of consumers' perceived value for the equipment of internet of things on pricing strategy, and it also analyze the impaction of consumers' perceived value for information service on pricing strategy, the research shows that the information providers should take different strategies based on the specific situations to maximize the profit on the information service market of the IOT.

Keywords: Internet of things; Individual pricing; Bundling pricing; Services pricing

\section{Introduction}

The 12th Five-Year Plan of China has clearly stated that Internet of Things should be developed to be a strategic emerging industry and promote its application in some important areas, highlighting that Internet of Things have been improved to the national strategic level. With the application of Internet of Things technology in the service industry, the pricing of information services cannot be avoided. What's more, the pricing of Internet of Things (IOT) is an important part to introduce information services into market. How to determine an appropriate pricing scheme which service providers, intermediaries and consumers are willing to accept, this makes pricing of Internet of things information service become a hotspot nowadays.

Why information services should be pricing for the IOT have got lots of attention in the literatures. Eva considers information as the main source for value proposition based on "economic value creation" theory, and uses the information law to prove his conclusion, then, proposing that pricing is an important step for introducing information service to the market. In addition (Eva \& Dieter, 2010), Elgar put IOT technology into use in the transportation, and analyses the users and 
businessmen's benefits, consequently he finds out that IOT can reduce transaction costs and market risks, then attempts to propose the appropriate market structure and pricing mechanisms which makes the business model successfully applied based on sensor service (Elgar, 2010). Jens applies the market design and pricing mechanism to the IOT, pointed the important participations in the internet of things service market, which includes consumers, intermediaries and information service providers. At the same time he gives the necessary steps to establish this kind of market (Jens \& Christoph, 2009). Wang and Yan analyse the application demands and industry driven effects of IOT, and indicates that the pricing of information service is the problem needed to be solved with the information service of IOT spread in use (Wang \& Yan, 2010). Chen confirms that information service of IOT is one of the most application forms of economic value, pointing the operators' role in the process of information service (Chen \& Han, 2010). Furthermore, Zhang points out that when promoting information services related to pricing, operators should integrate the advantages of resources and develops differentiated service (Zhang, 2010). IOT will provide location independent, interoperable, scalable, secure, and efficient access to a coordinated set of services, so we should adopt to a reasonable pricing to develop IOT. Theodore and Trakadas consider IOT are quickly gaining popularity due to the fact that can be used in a variety of application areas, the issue of privacy protection is as important as secure and reliable functioning of a network (Theodore \& Trakadas, 2009).

Some scholars make further studies for how to price in the IOT based on the above literatures, Michael and Markus apply IOT technology to the automobile insurance in order to ensure that the service pricing is based on actual driving risk, but do not represent the appropriate model. The result is mainly qualitative description but lacking of a quantitative support (Michael \& Markus, 2010). Christian and Martin concentrate on the technology applied into the supply chain, and puts forward a model of smart equipment costs from a quantitative point of view, treated the variation of technology prices, consumer utility and the fixed cost as parameters to assess income changes of providers, consumers and shippers, and finally provides pricing strategies for pricing makers (Christian \& Martin, 2010). Gerd, Kawsar, Fitton and Vasughi think an activity-aware smart object analyzes the data stream from its sensors, uses recognition algorithms to detect activities and events, and applies application-specific aggregation functions. So the discussion of usage-based pricing policies for smart products appears elsewhere (Gerd et al., 2010). Sun introduce a real case of Internet of Things called ZB IoT, It combines the Service Oriented Architecture (SOA) with EPC global standards in the system design, cited an example of how to pricing for the IOT (Sun \& Aersriten, 2010). Gao and Liang built a structural equation model of the internet of things, and the results showed that the consumers' potential demand for the internet of things was determined by their willingness and affordability, their willingness was determined by their perceived usefulness and perceived value (Gao \& Liang, 2012). Although it involves the pricing problem in his paper, it ignores the incomplete information conditions and market competition. 
From the above studies, we can see the articles applying the game theory to price information service are rare. This paper aims to analyze the strategic options and payoff function between information provider and intermediaries based on Stackelberg game. Firstly, we describe information service delivery method based on the Internet of Things specific function. Secondly, we calculate the consumer demand for the information service. Finally, we explain two kinds of strategic options by the game theory, and then discuss the optimal pricing method of information services based on profit maximization.

\section{The Basic Model}

\subsection{Model Setting}

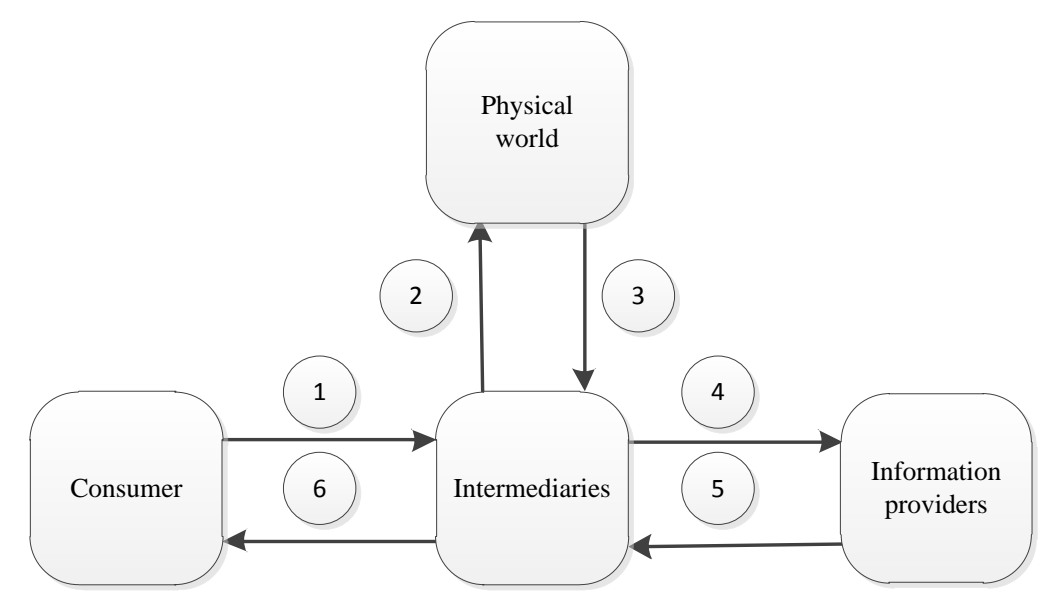

Figure 1 . The delivery routine of information service in the IOT

In the IOT market, it involves three entities when selling information services: information providers, intermediaries and consumers. Bundling is the first choice when selling sensor information to consumers. Throughout the whole economy activities, the consumer passively accepts the price. As shown in Figure 1, we can find out that the consumer firstly sends the information request to intermediaries, and then the intermediaries obtain basic information through the deployment of intelligent sensor node to the physical world. Furthermore, information providers integrate and process this physical information. Finally, the final information could be fed back to consumers by the verse routine. In the information services market, services fee that are charged by consumers is consist of two parts: expense paid to the information providers and that to intermediaries. Therefore, the relationship between intermediaries and information providers will affect the results of bundling pricing (Zhang \& Tang, 2008). In order to better analyze the problem, the participants are limited as follow:

\section{Consumer}

When consumers use the information provided by the IOT, the scene information has to be obtained by the perception equipment. Only when a wealth of information are aggregated 
together through a variety of sensors and labels for identification, perception, then coordinate with each other, can IOT provide a full real-time coverage and time-sensitive information services, and can consumers get information about location, environment at any place. In order to effectively solve the problem of information service pricing, this article adds the cost of the sensor equipment to elaborate the effect of information services pricing. It assumes that the consumers' reservation utility function is $V\left(R_{E}, p_{e}\right)$. Parameter $R_{E}$ is the consumers' evaluation value of the information services in an ideal condition, the value obeys a certain probability distribution, the density function is $f\left(R_{E}\right)$ and distribution function is $F\left(R_{E}\right)$. Parameter $p_{e}$ is the expenditure of perception equipment paid by consumers when enjoying the information services. Perception equipment and the required information have a complementary relationship with each other. In addition, parameter $R_{E}$ and parameter $p_{e}$ are not relevant with each other. Only when $R_{E} \geq p_{e}$ consumers will purchase the equipment, so the consumers reservation utility function can be expressed as: $V\left(R_{E}, p_{e}\right)=R_{E}-p_{e}$.

\section{Intermediaries}

Intermediaries provide market platform for connect information providers and consumers. Intermediaries are charged to consumers based on flow. This article supposes the price of per unit flow is $p_{i}$ and each size of information services (bytes) is also the same. It takes an average time $t$ on each information services for consumers. Profits from each service to intermediaries can be described as $p_{i} t$. Considering intermediaries providing distribution channels and marketing platforms, we assume that the marginal cost of transmission is zero.

\section{Information providers}

In the IOT Information services market, information providers supply $N(N \geq 2)$ kinds of different information services to consumers with zero marginal cost. There are two sales models: individual pricing and bundling pricing. In the individual pricing strategy, the unit price for information services is $p_{c}$. While in the bundling pricing, the price for the information service package is $p_{b}$. Information providers select a different sales model through the maximize profit principle, and thus making different prices for information services.

\subsection{Model Establishment}

When the information providers adopt different strategies, behaviors of consumers are different, so the demands of information services are also not similar with each other. When the information providers adopt bundling pricing strategy, for the intermediaries charge to consumers based on flow, if consumers spend a piece of information service, the fee paid to intermediaries is $p_{i} t$, and the consumer surplus can be expressed as: 


$$
C S_{c}=R_{E}-p_{e}-p_{c}-p_{i} t
$$

Only when $C S_{c} \geq 0$, consumers will spend on information services, so:

$$
R_{E} \geq p_{e}+p_{c}+p_{i} t
$$

Then the corresponding demand function is:

$$
d_{c}=\int_{p_{e}+p_{c}+p_{i} t}^{+\infty} f\left(R_{E}\right) d R_{E}=1-F\left(p_{e}+p_{c}+p_{i} t\right)
$$

When consumer needs $N$ pieces different information services, due to adopting bundling pricing strategy, customers need to purchase them in $N$ kinds of different markets to, so the total demand for information services is $D_{c}=N d_{c}$.

In order to facilitate the game analysis between intermediaries and information providers, we assume the consumers' evaluation of information services $R_{E}$ subjected to uniform distribution on $[0, R]$ in an ideal situation, so:

$$
D_{c}=N\left(1-\frac{p_{e}+p_{c}+p_{i} t}{R}\right)
$$

Under the condition of individual pricing strategy, when customers want to consume one or more pieces services in $N$ information services package, they must purchase the entire information services package, consumers' utility evaluation to the information service package will be affected by the correlation of service (Venkatesh \& Wagner, 2003), so the demand of service package market is not the sum of $N$ kinds of market requirements. The consumers' total evaluation of information service pack is $N R_{E}+\Delta V$. Compared with $N$ kinds of items for sale, $\Delta V$ represents the variation of consumers' evaluation under individual pricing strategy. When $\Delta V>0$, it means the value of the information service package is greater than the summation of every information service's value; if $\Delta V \leq 0$, it means the summation of every information service's value is not less than the value for bundling. The time consumers spending on service package is $N_{t}$, so the price paid to intermediaries is $N t p_{i}$, and the consumer surplus is $C S_{b}=N R_{E}+\Delta V p_{e}-p_{b}-N t p_{i}$. 
Only when $C S_{b}>0$, the customers would purchase the information service, so;

$$
R_{E} \geq \frac{p_{e}+N t p_{i}+p_{b}-\Delta V}{N}
$$

As a result, the demand for bundling pricing information service is:

$$
D_{b}=\int_{\frac{p_{e}+N t p_{i}+p_{b}-\Delta V}{N}}^{+\infty} f(x) d x=1-\frac{p_{e}+N t p_{i}+p_{b}-\Delta V}{N R}
$$

\section{Information Services Pricing of Providers Based on Stackelberg Game}

Under the circumstance of adopting different pricing models, in order to compare the difference of the information providers' profits, according to the literature 9, we can know the priority price maker will get first mover advantage in the information services market. Therefore, we analyze the profits difference for the information providers in this paper, it is the first to carry out pricing decisions for information providers, that is to say the information providers know the response function of intermediaries, while the intermediaries don't know the information provider's response function.

\subsection{Information Providers Profit under the Individual pricing Strategy}

At this point, the profit function of the information providers is:

$$
\pi_{s c}=p_{c} D_{c}=p_{c} N\left(1-\frac{p_{e}+p_{c}+p_{i} t}{R}\right)
$$

Because the intermediaries are charged fees based on the flow, in order to meet the demand, therefore the required time is written as $D_{c} t$, then, the expected profit intermediaries obtained is:

$$
\pi_{i c}=p_{i} D_{c} t=p_{i} t N\left(1-\frac{p_{e}+p_{c}+p_{i} t}{R}\right)
$$

Mathematically, it can be seen the formula (7) is quadratic function against $p_{c}$, formula ( 8 ) is also concave quadratic function against $p_{i}$. In order to maximize $\pi_{s c}$ and $\pi_{i c}$, we should make first-order derivative against $p_{c}$ for formula (7) and first-order derivative against $p_{i}$ for formula (8). According to the derivative to formula (8), we know that the intermediaries' response function of 
the information providers is:

$$
p_{i}=\frac{R-p_{e}-p_{c}}{2 t}
$$

Handling (9) into formula (7), we can know the profit function of the information providers is:

$$
\pi_{s c}=\frac{p_{c} N\left(R-p_{e}-p_{c}\right)}{2 R}
$$

Making formula (10) derivative against $p_{c}$, we can obtain the optimal prices respectively are:

$$
p_{c}=\frac{R-p_{e}}{2,} p_{i}=\frac{R-p_{e}}{4 t}
$$

And then, the profit function of the information providers is:

$$
\pi_{s c}=\frac{N\left(R-p_{e}\right)^{2}}{8 R}
$$

\subsection{Information Providers' Profit under the Bundling pricing Strategy}

So the profit function of the information providers is

$$
\pi_{s b}=p_{b} D_{b}=p_{b}\left(1-\frac{p_{e}+N t p_{i}+p_{b}-\Delta V}{N R}\right)
$$

At the same time, the profit function of the intermediaries is:

$$
\pi_{i b}=p_{i} D_{b} N t=p_{i} N t\left(1-\frac{p_{e}+N t p_{i}+p_{b}-\Delta V}{N R}\right)
$$

It is easy to see that formula (12) is concave to $p_{b}$, formula (13) is concave to $p_{i}$. In order to maximize the profit, we should make formula (12) first-order derivative against $p_{b}$ and formula (13) first-order derivative against $p_{i}$. 
According to the mentioned method, we obtain:

$$
p_{i}=\frac{N R-p_{e}-p_{b}+\Delta V}{2 N t}
$$

Substituting (15) into formula (13):

$$
\pi_{s b}=p_{b} \frac{N R-p_{e}-p_{b}+\Delta V}{2 N R}
$$

Making derivative against $p_{c}$ for formula (15), we can obtain the optimal prices are:

$$
p_{b}=\frac{N R-p_{e}+\Delta V}{2}, p_{i}=\frac{N R-p_{e}+\Delta V}{4 N t}
$$

At this point, the profit function of the information providers is:

$$
\pi_{s b}=\frac{\left(N R-p_{e}+\Delta V\right)^{2}}{8 N R}
$$

\section{Results Analysis and Numerical Simulation}

\subsection{Results Analysis}

Through the above analysis, we can find that the information providers adopt which kind of pricing methods depend on the relationship between $\pi_{b c}$ and zero in formula

$$
\pi_{b c}=\frac{\left(N R-p_{e}+\Delta V\right)^{2}}{8 N R}-\frac{N\left(R-p_{e}\right)^{2}}{8 R}=\frac{\left[(N-1) p_{e}+\Delta V\right]\left[2 N R-(N+1) p_{e}+\Delta V\right]}{8 N R}
$$

When we consider the price of the sensor device $p_{e}$ changing, while other variables are constant, making $\pi_{b c}$ equal to 0 , so, $p_{e 1}=-\frac{\Delta V}{N-1}, p_{\epsilon 2}=\frac{2 N R+\Delta V}{N+1}, p_{\varepsilon 2}-p_{\varepsilon 1}=\frac{2 N[R(N-1)+\Delta V]}{N^{2}-1}$.

PROPOSITION1. When the cost satisfies $R \geq p_{e}>\min \left[p_{e 1}, p_{e 2}\right]$, bundling pricing strategy is always better than individual pricing strategy. If $p_{e=} \min \left[p_{e 1}, p_{e 2}\right]$, there is no difference between individual pricing strategy and bundling pricing strategy, while $0 \leq p_{e}<\min \left[p_{\mathrm{e} 1}, p_{\mathrm{e} 2}\right]$, individual pricing strategy is better than bundling pricing strategy. 
When $\Delta V \geq 0$, obviously, we can get $\pi_{b c}>0$ from formula (17), that is to say at this point individual pricing strategy is better than bundling pricing strategy for the information providers. If $0>\Delta V>-R(N-1), p_{e 2}>p_{e 1}$, while $p_{e 2}>\left[\frac{2 N R-R(N-1)}{N+1}=R\right]$, the upper limit of $C$ is $R$, when $0<p_{e}<p_{e 1}$, then bundling pricing strategy is poorer than individual pricing strategy for the information providers. When it satisfies $p_{e 1} \leq p_{e}<R$, we could drop that the individual pricing strategy is not better than the bundling pricing strategy. While $-R N \leq \Delta V \leq-R(N-1), p_{e 2}<p_{e 1}$, $R \leq p_{e 1} \leq \frac{R N}{N-1}$, so the upper limit of $p_{e}$ is $R$, when $0<p_{e}<p_{e 2}$, the profit from bundling pricing strategy is inferior to the profit from individual pricing strategy. When $p_{\mathrm{e} 2} \leq p_{\mathrm{e}} \leq R$, the individual pricing strategy is not better than bundling pricing strategy. That means when the information providers make price decisions for information services in reality, they should consider the impact of the sensor device's price on pricing method, if the sensor device's price $p_{e}$ satisfies $R \geq p_{e}>\min \left[p_{e 1}, p_{e 2}\right]$, they should adopt bundling pricing strategy, otherwise, they should adopt individual pricing strategy.

Assuming that the variation of consumers' perception value of information services $\Delta V$ changes, other variables are constant, making $\pi_{b c}$ equal to 0 , we can get $\Delta V_{1}=-(N-1) p_{e}<0$, for the equation $\Delta V_{2}-\Delta V_{1}=2 N\left(p_{e}-R\right)<0$, so there is $\Delta V_{2}<0$.

PROPOSITION2: when the variation of consumers' perception value of information services $\Delta V>\Delta V_{1}$, the information providers should take bundling pricing strategy; if $\Delta V_{2}<\Delta V<\Delta V_{1}$, individual pricing strategy is better than bundling pricing strategy; while $\Delta V<\Delta V_{2}$, information providers should consider the sensor equipment pricing range to determine which

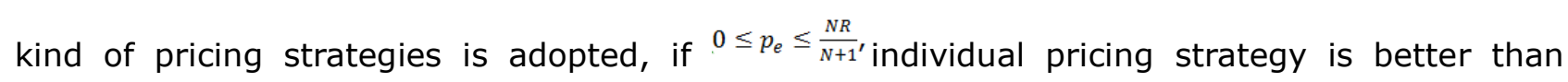
bundling pricing strategy; while $R \geq p_{e}>\frac{N R}{N+1}$, information producers should adopt bundling pricing strategy, this strengthens the conclusion when we consider the sensor device price $p_{e}$ changes.

If there is ${ }^{0 \leq p_{e} \leq \frac{N R}{N+1}^{\prime}}$, for $\Delta V_{2}+N R=(N+1) p_{e}-N R<0$, lower limit of consumers' retention effect is 0 . When $\Delta V>\Delta V_{1}$, the bundling pricing strategy is better than individual pricing strategy for the information providers, if there is $\Delta V \leq \Delta V_{1}$, bundling pricing strategy is not better than individual pricing strategy. When $R \geq p_{e} \geq \frac{N R}{N+1}$, no matter $\Delta V>\Delta V_{1}$ or $\Delta V<\Delta V_{2}$, the information providers should adopt bundling pricing strategy, but not individual pricing strategy in the IOT market. While $\Delta V_{2} \leq \Delta V \leq \Delta V_{1}$, bundling pricing strategy is worse than individual pricing strategy. This suggests when the information providers make price for information services in the IOT, they should adopt bundling pricing strategy for customers whose variation of perception value of information services is large; if the information providers want to take individual pricing strategy, 
the variation of customers evaluation toward information services should satisfy certain conditions. When the variation of customers' perception value of information services is small, which pricing strategy should be adopted depends on the sensor device price range.

\subsection{Numerical Simulation}

In view of the fact that the market of the IOT is in the preliminary developing stage now, market share is relatively smaller compared with other markets. What's more, its associated data is not easy to find. Therefore, this paper uses numerical simulation to verify the above conclusions. So we assume that the service package contain $N=6$ pieces of information services, in the ideal case, the consumers' evaluation value of the information services is $R=42$, the interval of the price $p_{e}$ of the sensor device is [15 41], the interval of the variation of consumers' perception value of information services $\Delta V$ is $\left[\begin{array}{lll}-250 & 10\end{array}\right]$. According to these figures and the above solution procedures, the information providers adopt bundling pricing strategy or individual pricing strategy respectively; the profits are shown in Figure 2, 3.

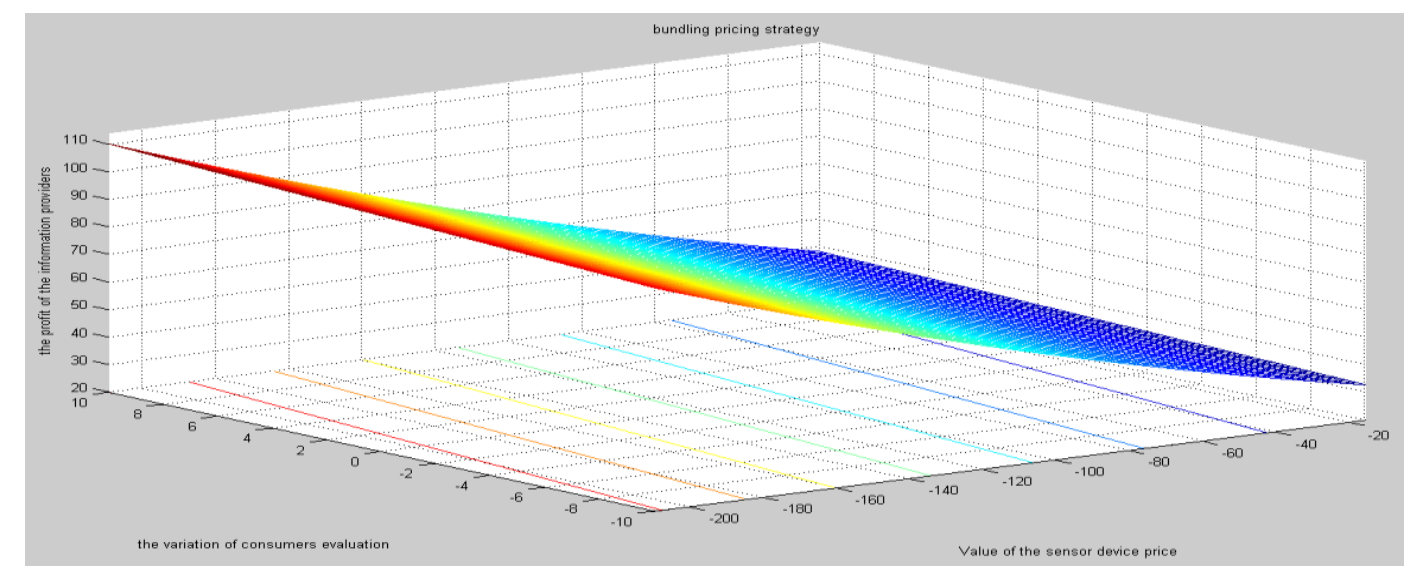

Figure 2. Profit $\pi_{s b}$ with bundling pricing strategy

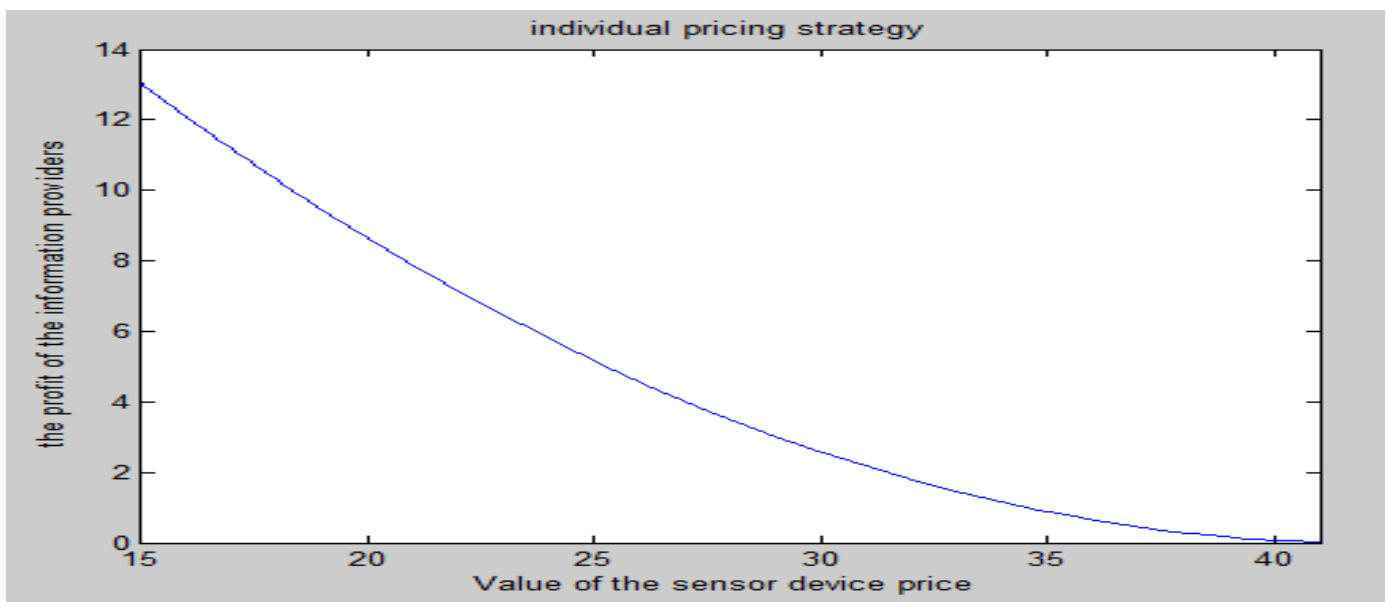

Figure 3. Profit $\pi_{s c}$ with individual pricing strategy 
From figure 2 and figure 3, we can see that when information providers adopt individual pricing strategy, the profit is only related to the sensor device price, but not related to the variation of consumers' perception value of information services, while they take bundling pricing strategy, the profit of information providers is related to the both. On the other hand, compared with the second group, the third, the fourth, the fifth, the seventh and the eighth group, we can obtain that when the variation of perception value of information services is the same, if the sensor device price is large, the information providers should adopt bundling pricing strategy, otherwise, they take individual pricing strategy. From the third group, the fourth group, the sixth, the seventh, the eighth, the ninth and the tenth group, we can obtain that when the sensor device price is the same, if the variation of perception value of information services is large, bundling pricing strategy should be adopted, but if it exceeds a certain minimum value, which strategy should be taken depends on the range of the sensor device price. The simulation results are entirely consistent with the conclusions in this article, that is to say the model is right.

\section{Concluding Remarks}

This paper researches the information providers should take different strategies based on the specific situations to maximize the profit on the information service market of the IOT. When the providers make price for the information service, in addition to continuously improve the quality of information service, it also devotes resources to tapping and understanding market information, such as the sensor device price, the variation of perception value of information services and so on, so as to create competitive advantage. This paper is just a preliminary model, it does not take into account the effect of mixed bundling, the author will make further research on this direction.

\section{Acknowledgment}

This study was supported by the Key National soft science research projects of China (2011GXS1B001), the key philosophy and social science research projects of Ministry of Education (11jzd032) and the soft science research projects of Hunan Province (2012ZK3036).

\section{References}

Chen, Z., \& Han, Z. (2010). Research information aggregation service mode and operator position in the Internet of Things. Mobile communication, 19:48-51.

Christian, D., \& Martin, B. (2009). Cost-Benefit Model for Smart Items in The Supply Chain. Communications of the Association for Information Systems, 5, 213-231.

Elgar, F. (2010). What is the Internet of Things . Business Processes \& Applications, 2, 1-27. 
Eva, B., \& Dieter, U. (2010). Business Models for the Internet of Things. Architecting the Internet of Things. UK, University of Cambridge Press, 252-279.

Future Internet Assembly. Bled Declaration on Future Internet. April 2008, www. future-internet.eu/index.php?id=47.

Gao, X.R., \& Liang, L. (2012). The Analysis of Influencing Factors of the Potential Demand for the Internet of Things Service Market-Based on an Investigation of Smart Home. East China Economic Management, 26(1), 33-38.

Gerd, K., Kawsar, F., Fitton, D. \& Vasughi, S. (2010). Smart Objects as Building Blocks for the Internet of Things. Internet Computing, 30-37.

Jens, B., \& Christoph, S. (2009). Initial Observations on Economics, Pricing, and Penetration of the Internet of Things Market [J]. Computer Communication Review, 39(2), 50-55. http://dx.doi.org/10.1145/1517480.1517491

Michael, C., \& Markus, L. (2010). The Internet of Things [J] . McKinsey Quarterly, 2, 1-9.

Sun, J., \& Aersriten (2010). Design and Implementation of an Enterprise Internet of Things. ASEA 2010, CCIS 117(2), 254-263.

Theodore \& Trakadas (2009). Securing wireless sensor networks towards a trusted "Internet of Things". Towards the Future Internet, 4:47-56.

Venkatesh, R., \& Wagner, K. (2003). Optimal bundling and pricing under a monopoly: Contrasting complements and substitutes from independently valued products[J]. Journal of Business, 76(4), 211-231. http://dx.doi.org/10.1086/367748

Wang, T. \& Yan, L. (2010). Application of the Internet of Things: demand oriented [J]. China Telecommunication Industry, 7(115), 44-45. http://dx.doi.org/10.1016/S1005-8885(09)60506-9

Zhang, Y. \& Tang, X. (2008). Study of online information goods bundle pricing [J]. Journal of Systems Engineering, 28(3), 331-337.

Zhang, Y. (2010). Discussion on the Business Model of Internet of Things [J] . Telecommunication science, 4, 6-11.

Journal of Industrial Engineering and Management, 2013 (www.jiem.org)

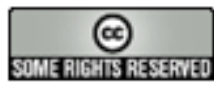

El artículo está con Reconocimiento-NoComercial 3.0 de Creative Commons. Puede copiarlo, distribuirlo y comunicarlo públicamente siempre que cite a su autor y a Intangible Capital. No lo utilice para fines comerciales. La licencia completa se puede consultar en http://creativecommons.org/licenses/by-nc/3.0/es/ 